\title{
Pulmonary thromboendarterectomy in chronic thromboembolic pulmonary hypertension: the Spanish experience
}

\author{
María Jesús López-Gude ${ }^{1}$, Isabel Blanco ${ }^{2,3,4}$, Victoria Benito-Arnáiz ${ }^{1}$, Manel Castellā ${ }^{5}$, \\ Pilar Escribano-Subías ${ }^{6,7}$, Clara Martin ${ }^{2,3}$, Joan Albert Barberàa ${ }^{2,3,4}$, José María Cortina-Romero ${ }^{1}$ \\ ${ }^{1}$ Department of Cardiac Surgery, Unidad Multidisciplinar de Hipertensión Pulmonar, European Reference Network for Respiratory Diseases (ERN- \\ lung), Hospital Universitario 12 de Octubre, Madrid, Spain; ${ }^{2}$ Department of Pulmonary Medicine, Hospital Clínic, Barcelona, Spain; ${ }^{3}$ Institut \\ d'Investigacions Biomèdiques August Pi i Sunyer (IDIBAPS), University of Barcelona; Barcelona, Spain; ${ }^{4}$ Biomedical Research Networking Center \\ in Respiratory Diseases (CIBERES), Madrid, Spain; ${ }^{5}$ Department of Cardiovascular Surgery, Cardiovascular Institute, Hospital Clínic, University \\ of Barcelona, Barcelona, Spain; ${ }^{6}$ Department of Cardiology, Unidad Multidisciplinar de Hipertensión Pulmonar, European Reference Network for \\ Respiratory Diseases (ERN-lung), Hospital Universitario 12 de Octubre, Madrid, Spain; ${ }^{7}$ Biomedical Research Networking Center in Cardiovascular \\ Diseases (CIBERCV), Madrid, Spain \\ Correspondence to: Victoria Benito-Arnáiz. Department of Cardiac Surgery, Hospital Universitario Hospital 12 de Octubre, Avda. de Córdoba s/n, \\ 28041 Madrid, Spain. Email: vbenitoa@gmail.com.
}

Background: Chronic thromboembolic pulmonary hypertension (CTEPH) can be cured by pulmonary endarterectomy (PEA). It is considered the best and only curable treatment option for patients with accessible lesions evaluated as optimal candidates. We describe the experience of the two reference centers in Spain, in order to reinforce the need for referring CTEPH patients to a specialized center to be assessed by a Multidisciplinary Expert Team.

Methods: We included a population of 338 patients who met the definition for CTEPH and underwent PEA between January 2007 and December 2019. The surgery was indicated in almost 60\% of patients assessed. Demographic, anthropometric, hemodynamic and echocardiographic features are listed for PEA patients. Immediate and one-year postoperative outcomes as well as overall mortality were analyzed.

Results: Mean age was $53.5 \pm 15.0$ years, $53.8 \%$ were men; a total of $68.5 \%$ were in WHO functional class III-IV; and most of them were in a preoperative hemodynamic condition: mean pulmonary arterial pressure (mPAP) was $46.5 \pm 13.1 \mathrm{mmHg}$ and mean pulmonary vascular resistance (PVR) was $764.5 \pm$ $392.8 \mathrm{dyn} \cdot \mathrm{s} \cdot \mathrm{cm}^{-5}$. PEA surgery was performed with cardiopulmonary bypass (CBP) and circulatory arrest, with very few complications [including neurological, postoperative reperfusion edema, extracorporeal membrane oxygenation (ECMO) implant and cardiac failure] and optimal postoperative results, where exercise capacity increased and mPAP and PVR values decreased significantly. Presence of persistent pulmonary hypertension $(\mathrm{PH})$ at the six-month right heart catheterization was evaluated. A $3.3 \%$ perioperative mortality was achieved. Overall, one-, three- and five-year survival rates were analyzed by Kaplan-Meier's method (94.8\%, 93.3\% and 90.5\% respectively), as well as for residual PH patients. Mortality risk factors were assessed.

Conclusions: Outstanding PEA results were seen in the immediate, one-year and long-term outcomes. The incidence of complications, including in-hospital mortality and long-term mortality were also below European rates.

Keywords: Pulmonary thromboendarterectomy; pulmonary hypertension (PH); pulmonary vascular resistance (PVR); residual pulmonary hypertension, operability

Submitted Oct 16, 2021. Accepted for publication Jan 10, 2022.

doi: $10.21037 /$ acs-2021-pte-18

View this article at: https://dx.doi.org/10.21037/acs-2021-pte-18 


\section{Introduction}

Chronic thromboembolic pulmonary hypertension $(\mathrm{CTEPH})$ is an infrequent evolution of acute pulmonary embolism (PE). Its prevalence varies from $0.57 \%$ to $9.1 \%$ (1); and several mechanisms behind the development of CTEPH have been suggested (2). It is a rare disease, associated with high morbidity and mortality when not treated $(3,4)$. It is still underdiagnosed but slowly starting to gain visibility. It should be diagnosed in its early phases so that CTEPH patients can be offered the best possible treatment $(1,5,6)$. Nowadays it is a potentially curable disease $(6,7)$, with pulmonary endarterectomy (PEA) being considered the treatment of choice in a high number of patients. This intervention pursues three main goals: (I) hemodynamic stability, reducing the effect of pulmonary hypertension $(\mathrm{PH})$ on the right ventricle by preventing right ventricular failure and secondary valvular disease; (II) respiratory stability, by improving ventilatory efficiency; and (III) improved exercise capacity $(1,5,8)$. PEA is a technically demanding operation, currently only performed in very few selected centers around the world; optimal results are associated with better patient selection, better perioperative care and greater surgical experience $(1,5,8)$. When performed in specialized centers, better results are found in terms of patient survival, functional class and exercise capacity due to the improvement of hemodynamics after the surgery (4,7,9-11). Furthermore, the ability of PEA to allow access to the lesions does not only depend on their anatomical distribution, but also on the surgeon's previous experience (2). There is a group of patients considered inoperable (around $35-40 \%$ of CTEPH patients) $(6,12)$, who have two other treatments options: balloon pulmonary angioplasty (BPA), and $\mathrm{PH}$-targeted medical therapy (MT) with riociguat (a stimulator of soluble guanylatecyclase enzyme) (13), which was approved in 2015 in Spain for patients with inoperable CTEPH or persistent PH after PEA.

National Spanish outcomes in CTEPH management are scarce due to the current decentralized model $(1,6)$. Currently, CTEPH can be treated in PH specialized centers or at general hospitals, where the decision of referring the patient for surgery is left to the treating doctor (1). However, all patients should be referred and carefully evaluated by a Multidisciplinary Expert Team, where every individual case is discussed, and the most suitable treatment is chosen. The Multidisciplinary Expert Team is made up of pulmonologists, cardiologists, radiologists and cardiac surgeons specialized in PEA (9). There are two $\mathrm{PH}$ specialized centers in Spain that bring together most CTEPH patients $(>60 \%)$, designated as Centros, Servicios y Unidades de Referencia del Sistema Nacional de Salud (CSUR): Hospital Clínic, Barcelona, and Hospital Universitario 12 de Octubre, Madrid $(5,14)$. These centers assess patients who belong to the corresponding health administrative area and those being referred from general hospitals (1). A recently published study shows that a low percentage of patients were referred to CSUR in Spain (61.4\%), which led to a lower rate of total PEA (30.7\%), and higher overall mortality (1). Furthermore, it is important to mention that, since 2007, a national observational registry of pulmonary hypertension (REHAP) has been running in order to evaluate national clinical management of CTEPH patients and its long-term outcomes in Spain $(1,6)$. Both specialized centers participate in the REHAP Registry and in the International CTEPH Registry (14).

Our objective is two-fold: (I) to investigate demographics, echocardiographic and hemodynamic characteristics of the 338 CTEPH patients who underwent surgery in CSUR Centers in Spain; and (II) to analyze surgical outcomes, immediately and up to one-year after the surgery. Inhospital and long-term mortality were analyzed. With this data, we want to reinforce our idea of changing the current model of CTEPH management in Spain.

\section{Methods}

\section{Inclusion criteria}

In the aforementioned centers, 578 patients between January $1^{\text {st }} 2007$ and December $31^{\text {st }} 2019$ met the definition of CTEPH, fulfilling therefore, the specific hemodynamic criteria for right heart catheterization (RHC): mean pulmonary artery pressure (mPAP) $\geq 25 \mathrm{mmHg}$, pulmonary vascular resistance (PVR) $\geq 3$ Wood units or $\geq 240$ dyn.s.cm ${ }^{-5}$ and pulmonary capillary wedge pressure (PCWP) $\leq 15$ $\mathrm{mmHg}$, or below this level but with documented exercise PH. Of the evaluated patients, 338 were considered operable, the remaining were deemed inoperable and given other treatment options. Moreover, all patients (operable and inoperable) showed perfusion defects in ventilation/ perfusion lung scintigraphy and CT angiography, consistent with CTEPH. They all received at least three months of anticoagulation treatment before the final diagnosis of CTEPH was given and continued receiving it long term. Data such as demographic and anthropometric parameters, 


\begin{tabular}{|c|c|}
\hline Variables & Mean \pm SD or $n(\%)$ \\
\hline \multicolumn{2}{|c|}{ Demographic and anthropometric } \\
\hline Age (years) & $53.5 \pm 15.0$ \\
\hline Male gender & $182(53.8)$ \\
\hline $\mathrm{BMI}\left(\mathrm{kg} / \mathrm{m}^{2}\right)$ & $28.0 \pm 5.2$ \\
\hline Systemic hypertension & $91(35.0)$ \\
\hline DM & $25(9.6)$ \\
\hline Current/past smoking habit & $105(40.4)$ \\
\hline Coronary artery disease & $16(6.2)$ \\
\hline Cancer history & $25(9.7)$ \\
\hline Hypercoagulability & 115 (34.0) \\
\hline PE history & $290(85.8)$ \\
\hline DVT & $153(45.3)$ \\
\hline \multicolumn{2}{|l|}{ Clinical } \\
\hline WHO I-II & $101(31.5)$ \\
\hline WHO III-IV & $220(68.5)$ \\
\hline 6MWD (m) & $399.4 \pm 123.4$ \\
\hline NT-proBNP (mg/dL) & $1,403.3 \pm 2,034.9$ \\
\hline \multicolumn{2}{|c|}{ Hemodynamic and echocardiographic } \\
\hline mPAP $(\mathrm{mmHg})$ & $46.5 \pm 13.1$ \\
\hline PVR (dyn.s $\cdot \mathrm{cm}^{-5}$ ) & $764.5 \pm 392.8$ \\
\hline RAP $(\mathrm{mmHg})$ & $9.1 \pm 5.4$ \\
\hline PCWP (mmHg) & $10.3 \pm 4.0$ \\
\hline Cardiac index $\left(\mathrm{L} / \mathrm{min} / \mathrm{m}^{2}\right)$ & $2.30 \pm 0.59$ \\
\hline TAPSE (mm) & $17.4 \pm 4.3$ \\
\hline Pericardial effusion & $32(12.8)$ \\
\hline
\end{tabular}

BMI, body mass index; DM, diabetes mellitus; PE, pulmonary embolism; DVT, deep vein thrombosis; WHO, World Health Organization; 6MWD, six-minute walk distance; NT-proBNP, $\mathrm{N}$-terminal pro B-type natriuretic peptide; mPAP, mean pulmonary arterial pressure; PVR, pulmonary vascular resistance; RAP, right atrial pressure; PCWP, pulmonary capillary wedge pressure; SD, standard deviation; TAPSE, tricuspid annular plane systolic excursion.

PH clinical characteristics and supplementary diagnostic tests parameters (echocardiographic and RHC variables) (Table 1) were obtained from routine medical visits.

\section{PEA selection}

Most patients will benefit from PEA surgery, but the selection criteria remain subjective to each multidisciplinary team. It is based on several factors, such as lesion accessibility (anatomic distribution-assessed by CT angiography-and the cardiac surgeon's expertise), severity of the patient's disease including symptoms and hemodynamic status (severity of $\mathrm{PH}$ and right heart dysfunction) and presence of co-morbidities, including long-term expectations $(1,12)$. Therefore, the selection of candidates for surgery depends on the combination of accessible surgical disease and the severity of $\mathrm{PH}$ and right ventricular dysfunction, but neither the severity of right ventricular dysfunction nor the value of PVR will exclude a patient from surgical consideration, however, the PEA and postoperative care are made more challenging. In terms of accessibility, if the disease is in the main, lobar or segmental pulmonary artery branches, endarterectomy is feasible; and it is only performed in the most expert centers even if subsegmental arteries are primarily affected $(1,2,5,6,12)$. Nevertheless, distal segmental disease is much more difficult to remove and renders the patient inoperable $(7,12,15)$. The decision to operate was always made in a meeting of members of the Multidisciplinary Pulmonary Hypertension Team.

\section{Surgical technique and follow-up}

PEA was performed in accordance with the University of California's protocol (San Diego, USA) (12). Surgical approach was through median sternotomy, and performed with full cardiopulmonary bypass, aortic cross-clamping and deep, hypothermia. Endarterectomy was performed during ten-minute periods of circulatory arrest, followed by five-minute reperfusion lapses. The extracted material during the PEA was grouped according to histopathological prognostic value established by the University of California group (16): type 1, fresh thrombus in the main-lobar pulmonary arteries; type 2, intimal thickening and fibrosis proximal to the segmental arteries, with no thrombus; type 3 , disease within distal segmental arteries only; and type 4, distal arteriolar vascular disease. In-hospital mortality and all causes of death were collected. Postoperative complications were described as presence of reperfusion edema (postoperative respiratory failure causing hypoxia, accompanied by pulmonary infiltrates on chest X-ray in 
some of the surgical areas, occurring in the first 72 hours after PEA, and needing mechanical ventilation for more than 96 hours), cardiac failure, need for extracorporeal membrane oxygenation (ECMO), neurological complications and residual $\mathrm{PH}$. Both the definition of residual $\mathrm{PH}$ and the time for diagnosis are controversial; some authors establish it according to mPAP values and others to PVR values. In our study, residual $\mathrm{PH}$ was defined as having mPAP $>25 \mathrm{mmHg}$ at rest, and clinically relevant $\mathrm{PH}$ as having PVR $>400 \mathrm{dyn} \cdot \mathrm{s} \cdot \mathrm{cm}^{-5}$ (in the six-month RHC). All patients remained on long-term anticoagulant therapy. Follow-up time was at least one year.

\section{Statistical analysis}

Continuous variables were expressed as a mean \pm standard deviation, or as a median with interquartile range (IQR) when not normally distributed. Quantitative variables were analyzed using the Student's $t$-test or Mann-Whitney $\mathrm{U}$-test. Comparisons were made using the paired $t$-test or ANOVA. Categorical variables were expressed as frequencies, $\mathrm{n}(\%)$ and compared using the Chi-square, Fisher's exact test or McNemar's for paired samples. All $\mathrm{P}$ values were two-sided, with a $\mathrm{P}$ value $<0.05$ being considered statistically significant. Preoperative, intraoperative, and postoperative variables were analyzed to evaluate if they statistically correlated with in-hospital mortality using logistic regression; those that were available in less than a $70 \%$ of patients were excluded [such as $\mathrm{N}$-terminal pro B-type natriuretic peptide (NT-proBNP) which was firstly measured in 2009 in one of the two expert centers]. Multivariable logistic regression analysis was performed for variables considered risk factors, to calculate their relative risk and $95 \%$ confidence interval (CI). The univariate Cox regression model was used to evaluate proportional hazards for mortality, and those that revealed a $\mathrm{P}<0.05$ significance were included in the multivariable analysis (again NT-proBNP was not included in this Cox analysis). The proportional hazards assumptions were checked using scaled Shoenfeld residual, using both hypothesis testing and graphical methods. The linearity assumptions were checked by plotting the Martingale residuals against continuous covariates. Survival curves were calculated using the Kaplan-Meier method and compared using the log rank test. The study date of entry was defined as the date of the first diagnostic RHC. The statistical analysis was performed using SPSS version 17 (SPSS Inc., Chicago, IL, USA) and $\mathrm{R}$ software version 4.1.1.

\section{Results}

\section{Study population and situation at diagnosis}

The study population included a group of 338 patients with CTEPH who met the inclusion criteria and underwent surgery between January $1^{\text {st }} 2007$ and December $31^{\text {st }} 2019$; constituting $58.5 \%$ of the total assessed patients across the two specialized centers. Clinical and hemodynamic characteristics at diagnosis of all patients are listed in Table 1; mean age was $53.5 \pm 15.0$ years, $53.8 \%$ were men and most of patients had severe clinical disease [ $68.5 \%$ were in World Health Organisation (WHO) functional class III-IV] and a severe hemodynamic condition (with a mPAP of $46.5 \pm$ $13.1 \mathrm{mmHg}$ and 80 patients $(23.7 \%)$ had previous PVR $\left.>1,000 \mathrm{dyn} \cdot \mathrm{s} \cdot \mathrm{cm}^{-5}\right)$.

\section{Immediate and one-year outcomes}

\section{PEA immediate postoperative outcomes}

In patients who underwent PEA, mean CBP time was $215.8 \pm 47.0$ minutes, mean cross-clamp time was $113.1 \pm$ 24.6 minutes, with a mean time of circulatory arrest of $42.8 \pm 14.5$ minutes. Complete PEA was performed in $95.5 \%$ and concomitant surgery in $70(20.6 \%)$ patients (Table 2$)$. According to the San Diego classification system of the biological material extracted, $27.9 \%$ was classified as type 1 , $49.8 \%$ as type 2 and $22.3 \%$ as type 3 . Complications in postoperative care were registered; the global in-hospital mortality was $3.3 \%$, postoperative reperfusion edema occurred in $14.2 \%$ of patients, cardiac failure in $6.5 \%$ and ECMO was required in 25 patients (7.4\%), 18 of which were veno-venous and 7 were veno-arterial. Additionally, postoperative neurological complications occurred in 5.9\% of patients, most of which were temporary (5.3\%) but in two cases $(0.6 \%)$ caused permanent damage. Median intensive care unit (ICU) stay was 6 (IQR 8) days; and in-hospital stay was 14 (IQR 12) days (Table 2). Patients' preoperative, intraoperative and postoperative characteristics potentially associated with greater in-hospital mortality were assessed by logistic regression univariate analysis; the items found to be risk factors for in-hospital mortality are specified in Table 3. Following multivariable analysis, only previous PVR $>1,000$ dyn $\cdot s \cdot \mathrm{cm}^{-5}$ remained an independent risk factor for in-hospital mortality $(\mathrm{P}=0.010)$ (Table 3).

\section{PEA one-year clinical and hemodynamic outcomes}

One-year outcomes in PEA patients were assessed: both exercise capacity [6-minute walk distance (6MWD)] and 


\begin{tabular}{|ll}
$\begin{array}{l}\text { Table } 2 \text { Intraoperative characteristics and immediate postoperative } \\
\text { complications }\end{array}$ & $\begin{array}{l}\text { Mean } \pm \text { SD or } n(\%) \\
\text { or median [IQR] }\end{array}$ \\
\hline Variables & $215.8 \pm 47.0$ \\
\hline CPB time (min) & $113.1 \pm 24.6$ \\
\hline Cross-clamp time (min) & $42.8 \pm 14.5$ \\
\hline Circulatory arrest (min) & $323(95.5)$ \\
\hline Complete PEA & $70(20.6)$ \\
\hline Concomitant surgery & \\
\hline San Diego anatomo-surgical classification & $89(27.9)$ \\
\hline Type 1 & $159(49.8)$ \\
\hline Type 2 & $71(22.3)$ \\
\hline Type 3 & $6[4-12.8]$ \\
\hline ICU stay (days) & 14 [9-21] \\
\hline In-hospital stay (days) & $11(3.3)$ \\
\hline In-hospital mortality & $48(14.2)$ \\
\hline Reperfusion edema & $22(6.5)$ \\
\hline Cardiac failure & $25(7.4)$ \\
\hline ECMO & $20(5.9)$ \\
\hline Neurological complications & \\
\hline CBP, cardiopumonary bypass; PEA & \\
\hline
\end{tabular}

CBP, cardiopulmonary bypass; PEA, pulmonary endarterectomy: ICU, Intensive Care Unit; IQR, interquartile range; ECMO, extracorporeal membrane oxygenator; SD, standard deviation.

clinical functional class (WHO classification) improved, and NT-proBNP values decreased significantly. Moreover, hemodynamic and echocardiographic features improved significantly after surgery: mPAP, RAP and PVR values decreased, and statistical differences were found between preoperative and postoperative figures. Additionally, both cardiac index and tricuspid annular plane systolic excursion (TAPSE) values improved after PEA (Table 4).

\section{Residual PH after PEA}

For patients whose data was available $(\mathrm{n}=260), 130(50 \%)$ had persistent residual PH (mPAP $>25 \mathrm{mmHg})$; and clinically relevant residual PH (PVR $>400$ dyn $\cdot \mathrm{s} \cdot \mathrm{cm}^{-5}$ ) was seen in $59(22.7 \%)$ patients. Mean preoperative PVR in the latter group of patients was $844.2 \pm 314.9 \mathrm{dyn} \cdot \mathrm{s} \cdot \mathrm{cm}^{-5}$, higher than the rest of the patients.

\section{PEA survival}

During a mean follow-up of $38.5 \pm 27.3$ months, there were a total of 27 deaths. One-, three- and five-year survival rates from diagnosis were $94.8 \%, 93.3 \%$ and $90.5 \%$, respectively (Figure 1). Residual PH mortality was also evaluated; one-, three-, and five-year survival rates were $94.8 \%, 87.8 \%$ and $87.8 \%$, respectively, and were slightly lower in those patients with normalized PVR after surgery, although, survival rates equalized six years after surgery (log-rank 0.004) (Figure 2). Univariate Cox regression was performed to evaluate mortality risk factors during follow-up (Table 5). For multivariable Cox analysis, only a higher 6MWD $(\mathrm{P}=0.009)$, therefore better physical capacity and higher cardiac output (by every $0.5 \mathrm{~L} / \mathrm{min}$ increase) $(\mathrm{P}=0.033)$, remained an independent protective factor for mortality during follow-up (Table 5).

\section{Discussion}

CTEPH is a rare disease, with 8.9 cases per million inhabitants in Spain, despite it being thought to be underdiagnosed $(1,2)$. Our study provides characteristics at diagnosis from 338 operable patients assessed in the CSUR expert centers for the management of complex $\mathrm{PH}$, and outcomes after PEA including survival rates. Clinical guidelines (7) and consensus documents $(1,2,17)$ establish the need to refer these patients to Multidisciplinary Expert Teams with expert surgeons to treat CTEPH. In fact, surgery should not be ruled out in any patient before being evaluated by an expert team. In the International CTPEH Registry (11), up to $43 \%$ of the patients evaluated were not considered candidates for surgery. A proportion of $75.7 \%$ of patients in the Spanish Registry (REHAP) did not undergo surgery (1). However, as previously shown, in CSUR specialized centers, almost $60 \%(58.5 \%$ of 578 patients) underwent surgery, after being evaluated by a Multidisciplinary Expert Team.

Analysis of the data revealed that the results of our series are excellent, despite severe previous hemodynamic condition. In our study population, $68.5 \%$ were classified as WHO III-IV functional class at the time of surgery $(5.3 \%$ as WHO IV), and mPAP was $46.5 \pm 13.1 \mathrm{mmHg}$ and mean PVR was $764.5 \pm 392.8$ dyn $\cdot \mathrm{s} \cdot \mathrm{cm}^{-5}$. Furthermore, PEA surgical times were similar to international specialized centers, including a mean total arrest time of $42.8 \pm 14.5$ minutes 


\begin{tabular}{|c|c|c|c|c|}
\hline \multirow{2}{*}{ Risk factor } & \multicolumn{2}{|c|}{ Univariate analysis } & \multicolumn{2}{|c|}{ Multivariate analysis } \\
\hline & $\mathrm{RR}[95 \% \mathrm{Cl}]$ & $P$ & $\mathrm{RR}[95 \% \mathrm{Cl}]$ & $P$ \\
\hline WHO functional class IV & $10[23-47]$ & 0.001 & & \\
\hline RAP & $1.1[1.0-1.2]$ & 0.034 & & \\
\hline PVR $>1,000$ dyn $\cdot \mathrm{s} \cdot \mathrm{cm}^{-5}$ & 8 [2-27] & 0.009 & 10.5 [1.8-85.0] & 0.010 \\
\hline Reperfusion edema & 11 [4-99] & $<0.001$ & & \\
\hline Cardiac failure & $12[2-57]$ & 0.001 & & \\
\hline ECMO & $22[5-150]$ & $<0.001$ & & \\
\hline
\end{tabular}

WHO, World Health Organization; RAP, right atrial pressure; PVR, pulmonary vascular resistance; ECMO, extracorporeal membrane oxygenator; RR, relative risk; $\mathrm{Cl}$, confidence interval.

Table 4 Comparison between preoperative and postoperative patient characteristics after PEA

\begin{tabular}{|c|c|c|c|}
\hline Variables & Preoperative & One-year outcomes & P (bilateral) \\
\hline \multicolumn{2}{|l|}{ WHO classification, n (\%) } & & $<0.001$ \\
\hline I-II & $76(27.8)$ & \multicolumn{2}{|l|}{$261(95.6)$} \\
\hline III-IV & $197(72.2)$ & \multicolumn{2}{|l|}{$12(4.4)$} \\
\hline NT-proBNP $(\mathrm{mg} / \mathrm{dL})$, mean $\pm \mathrm{SD}$ & $1315.3 \pm 1,589.5$ & $288.6 \pm 322.7$ & $<0.001$ \\
\hline \multicolumn{4}{|c|}{ Hemodynamic and echocardiographic, mean \pm SD } \\
\hline mPAP $(\mathrm{mmHg})$ & $46.4 \pm 13.0$ & $27.3 \pm 10.3$ & $<0.001$ \\
\hline PVR (dyn $\left.\cdot s \cdot \mathrm{cm}^{-5}\right)$ & $757.8 \pm 375.2$ & $329.0 \pm 477.5$ & $<0.001$ \\
\hline Cardiac index & $2.30 \pm 0.59$ & $2.70 \pm 0.57$ & $<0.001$ \\
\hline TAPSE (mm) & $17.8 \pm 4.3$ & $16.7 \pm 3.5$ & 0.034 \\
\hline
\end{tabular}

and mean CBP of $215.8 \pm 47.0$ minutes; despite concomitant surgery being performed in a group of $70(20.6 \%)$ patients. Overall perioperative mortality was $3.3 \%$, similar to the largest series reported in the literature by international specialized centers (8,17-19), and below mean European rates (7); placing Spanish CSUR CTEPH Centers in an outstanding international position. Our success is due to the expertise of our surgeons and the procedural protocol developed by the Multidisciplinary Expert Team. Surgical outcomes and survival in both centers in Madrid and Barcelona have improved as there has been a gradual increase in the number of surgical candidates and the team acquired more experience $(2,20,21)$. The accessibility of lesions varies according to the level of experience of the 


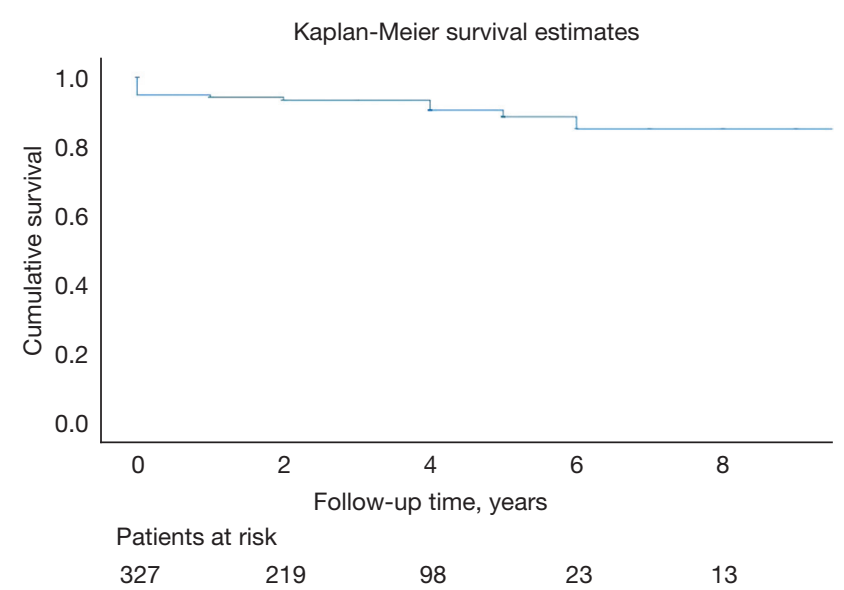

Figure 1 Kaplan-Meier survival estimates, including in-hospital mortality.

surgical team. In most experienced teams, the percentage of patients with segmental branch involvement (San Diego type 3 ) increases over time, constituting $22.3 \%$ of total operated patients in CSUR centers $(12,19)$. It is remarkable that our series registers very few cases of neurological complications (5.9\%), most of them being transitory after PEA intervention, despite not performing PEA with continuous cerebral perfusion as suggested by other groups (22). Other postoperative complications were also rare, such as cardiac failure (6.5\%), or ECMO implant, where only $2.1 \%$ of PEA patients required veno-arterial ECMO and $5.3 \%$ veno-venous ECMO. Reperfusion injury is inherent to PEA and its incidence ranges from $5 \%$ to $20 \%$, as published by different series $(10,12,14)$. According to our outcomes, reperfusion injury was recorded in $14.2 \%$ of patients. It is noteworthy that after PEA, hemodynamic and echocardiographic outcomes improved significantly; patients' exercise capacity (6MWD), cardiac index and TAPSE index also improved; and NT-proBNP values decreased, as did mPAP and RAP values. Two important features must be highlighted; on one hand, PVR values decreased significantly (preoperative $757.8 \pm 375.2 \mathrm{vs}$. postoperative $329.0 \pm 477.5 \mathrm{dyn} \cdot \mathrm{s} \cdot \mathrm{cm}^{-5}, \mathrm{P}<0.001$ ), although $50 \%$ of patients had residual $\mathrm{PH}(\mathrm{mPAP}>25 \mathrm{mmHg})$, while only $22.7 \%$ of patients had clinically relevant residual $\mathrm{PH}$ in RHC (PVR > 400 dyn $\cdot \mathrm{s} \cdot \mathrm{cm}^{-5}$ ) performed at six months after PEA, according to international registries $(12,14,23)$. All such patients were individually assessed in order to choose further treatment options; $16.7 \%$ were candidates for BPA after surgery, and the rest were evaluated to receive targeted-MT such as riociguat, after its approval in Spain

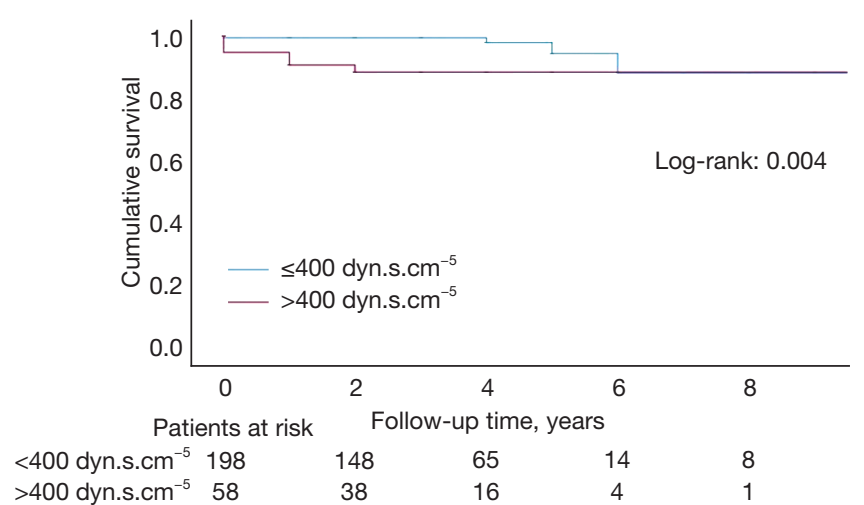

Figure 2 Kaplan-Meier survival estimates in residual $\mathrm{PH}$ patients and those with no residual $\mathrm{PH}$. PH, pulmonary hypertension.

in 2015. Moreover, in our series we performed a six-month follow-up visit for two reasons: hemodynamic changes immediately following surgery will affect estimates of PVR, and also because the prevalence of residual $\mathrm{PH}$ increases over time when the cause is distal vascular disease (24).

In this study, potential factors associated with greater in-hospital mortality were also investigated. Previous PVR $>1,000$ dyn $\cdot \mathrm{s} \cdot \mathrm{cm}^{-5}$ was a risk factor for in-hospital mortality in both the univariate and multivariable logistic regression analysis, similarly to other published series $(12,19)$. Regarding follow-up, multiple mortality risk factors were found in the univariate Cox analysis. In the multivariable analysis, only longer preoperative distances in the sixminute walking test and high cardiac output were found to be independent protective factors for mortality, most likely due to better previous physical and hemodynamic conditions. Kaplan-Meier survival analysis showed outstanding survival rates of $94.8 \%, 93.3 \%$ and $90.5 \%$ in the one-, three- and five-year follow-up, respectively, similar to results reported in other major studies $(12,15,19,23)$. For residual $\mathrm{PH}$ patients, survival rates were only slightly lower (94.8\%, $87.8 \%$ and $87.8 \%$ at one-, three- and five-year follow-up, respectively) when compared with those patients whose PVR normalized after surgery (log-rank 0.004).

Excellent follow-up was achieved in $98.8 \%$ of patients; only 4 patients did not attend any check-up. A significant limitation when interpreting the results is that the data were collected by two different centers, where some data may be missing or inconclusive.

This study highlights the favorable results obtained in CSUR centers for CTEPH in Spain, with Multidisciplinary Expert Teams that evaluate patients according to their 


\begin{tabular}{|c|c|c|c|c|}
\hline Variables & $\mathrm{HR}(95 \% \mathrm{Cl})$ & $\mathrm{P}$ & $\mathrm{HR}(95 \% \mathrm{Cl})$ & $\mathrm{P}$ \\
\hline \multicolumn{5}{|l|}{ Demographic } \\
\hline Age & $1.01(0.99-1.07)$ & 0.156 & & \\
\hline BMI & $0.95(0.83-1.08)$ & 0.414 & & \\
\hline DM & $0.04(0.00-2.91)$ & 0.488 & & \\
\hline Cancer history & $3.60(1.57-10.58)$ & 0.009 & & \\
\hline Current/past smoking habit & $1.26(0.45-3.51)$ & 0.657 & & \\
\hline \multicolumn{5}{|l|}{ Clinical } \\
\hline WHO functional class III-IV & $2.00(0.45-9.00)$ & 0.214 & & \\
\hline 6MWD (increase by 30 m) & $0.70(0.65-0.85)$ & $<0.001$ & $0.80(0.75-0.94)$ & 0.009 \\
\hline \multicolumn{5}{|l|}{ Hemodynamic } \\
\hline RAP (increase by $10 \mathrm{mmHg}$ ) & $0.99(0.40-2.35)$ & 0.973 & & \\
\hline mPAP (increase by $10 \mathrm{mmHg}$ ) & $1.15(0.77-1.76)$ & 0.475 & & \\
\hline PVR (increase by $200 \mathrm{dyn} \cdot \mathrm{s} \cdot \mathrm{cm}^{-5}$ ) & $1.34(1.10-1.60)$ & 0.001 & & \\
\hline CO (increase by $0.5 \mathrm{~L} / \mathrm{min}$ ) & $0.62(0.48-0.82)$ & 0.001 & $0.61(0.39-0.96)$ & 0.033 \\
\hline
\end{tabular}

specific case and recommend the most appropriate treatment.

\section{Conclusions}

A cohort of 338 patients out of 578 patients diagnosed with CTEPH from 2007 to 2019, underwent PEA at two Spanish CTEPH specialized centers. Surgical patients had outstanding survival rates at one-, three- and fiveyear follow-up, and a high in-hospital survival rate for PEA patients was confirmed. Pulmonary endarterectomies were performed within short CBP and circulatory arrest times, with very few complications (including neurological, postoperative reperfusion edema, ECMO implant and cardiac failure) and good one-year results, where exercise capacity increased, and mPAP and PVR values significantly decreased. Mortality risk factors were also evaluated. Due to the optimal results obtained in CSUR centers, we reinforce our statement that all patients should be referred for operability assessment at specialized centers established by international guidelines.

\section{Acknowledgments}

Funding: None.

\section{Footnote}

Conflicts of Interest: The authors have no conflicts of interest to declare. 
Open Access Statement: This is an Open Access article distributed in accordance with the Creative Commons Attribution-NonCommercial-NoDerivs 4.0 International License (CC BY-NC-ND 4.0), which permits the noncommercial replication and distribution of the article with the strict proviso that no changes or edits are made and the original work is properly cited (including links to both the formal publication through the relevant DOI and the license). See: https://creativecommons.org/licenses/by-nc-nd/4.0/.

\section{References}

1. Martínez-Santos P, Velázquez-Martín MT, Barberá JA, et al. Chronic thromboembolic pulmonary hypertension in Spain: a decade of change. Rev Esp Cardiol (Engl Ed) 2021;74:384-92.

2. López Gude MJ, Pérez de la Sota E, Forteza Gil A, et al. Pulmonary thromboendarterectomy in 106 patients with chronic thromboembolic pulmonary hypertension. Arch Bronconeumol 2015;51:502-8.

3. McNeil K, Dunning J. Chronic thromboembolic pulmonary hypertension (CTEPH). Heart 2007;93:1152-8.

4. Hoeper MM, Mayer E, Simonneau G, et al. Chronic thromboembolic pulmonary hypertension. Circulation 2006;113:2011-20.

5. Condliffe R, Kiely DG, Gibbs JS, et al. Improved outcomes in medically and surgically treated chronic thromboembolic pulmonary hypertension. Am J Respir Crit Care Med 2008;177:1122-7.

6. Escribano-Subias P, Blanco I, López-Meseguer M, et al. Survival in pulmonary hypertension in Spain: insights from the Spanish registry. Eur Respir J 2012;40:596-603.

7. Galiè N, Humbert M, Vachiery JL, et al. 2015 ESC/ERS Guidelines for the diagnosis and treatment of pulmonary hypertension: The Joint Task Force for the Diagnosis and Treatment of Pulmonary Hypertension of the European Society of Cardiology (ESC) and the European Respiratory Society (ERS): Endorsed by: Association for European Paediatric and Congenital Cardiology (AEPC), International Society for Heart and Lung Transplantation (ISHLT). Eur Heart J 2016;37:67-119.

8. Ghofrani HA, Hoeper MM, Halank M, et al. Riociguat for chronic thromboembolic pulmonary hypertension and pulmonary arterial hypertension: a phase II study. Eur Respir J 2010;36:792-9.

9. López Gude MJ, Pérez de la Sota E, Pérez Vela JL, et al. Pulmonary endarterectomy outputs in chronic thromboembolic pulmonary hypertension. Med Clin (Barc) 2017;149:1-8.

10. Jenkins D, Madani M, Fadel E, et al. Pulmonary endarterectomy in the management of chronic thromboembolic pulmonary hypertension. Eur Respir Rev 2017;26:160111.

11. Mayer E, Jenkins D, Lindner J, et al. Surgical management and outcome of patients with chronic thromboembolic pulmonary hypertension: results from an international prospective registry. J Thorac Cardiovasc Surg 2011;141:702-10.

12. Madani MM. Surgical Treatment of Chronic Thromboembolic Pulmonary Hypertension: Pulmonary Thromboendarterectomy. Methodist Debakey Cardiovasc J 2016;12:213-8.

13. Ghofrani HA, D'Armini AM, Grimminger F, et al. Riociguat for the treatment of chronic thromboembolic pulmonary hypertension. N Engl J Med 2013;369:319-29.

14. Pepke-Zaba J, Delcroix M, Lang I, et al. Chronic thromboembolic pulmonary hypertension (CTEPH): results from an international prospective registry. Circulation 2011;124:1973-81.

15. Rahnavardi M, Yan TD, Cao C, et al. Pulmonary thromboendarterectomy for chronic thromboembolic pulmonary hypertension : a systematic review. Ann Thorac Cardiovasc Surg 2011;17:435-45.

16. Thistlethwaite PA, Mo M, Madani MM, et al. Operative classification of thromboembolic disease determines outcome after pulmonary endarterectomy. J Thorac Cardiovasc Surg 2002;124:1203-11.

17. Kim NH, Delcroix M, Jenkins DP, et al. Chronic thromboembolic pulmonary hypertension. J Am Coll Cardiol 2013;62:D92-9.

18. Mayer E. Surgical treatment of chronic thromboembolic pulmonary hypertension. Swiss Med Wkly 2006;136:491-7.

19. Kim NH, Delcroix M, Jais X, et al. Chronic thromboembolic pulmonary hypertension. Eur Respir J 2019;53:1801915.

20. Blázquez JA, Escribano P, Pérez E, et al. Chronic thromboembolic pulmonary hypertension: surgical treatment with thromboendarterectomy. Arch Bronconeumol 2009;45:496-501.

21. Coronel ML, Chamorro N, Blanco I, et al. Medical and surgical management for chronic thromboembolic pulmonary hypertension: a single center experience. Arch Bronconeumol 2014;50:521-7.

22. Vuylsteke A, Sharples L, Charman G, et al. Circulatory 
arrest versus cerebral perfusion during pulmonary endarterectomy surgery (PEACOG): a randomised controlled trial. Lancet 2011;378:1379-87.

23. Jenkins D. Pulmonary endarterectomy: the potentially curative treatment for patients with chronic

Cite this article as: López-Gude MJ, Blanco I, Benito-Arnáiz V, Castellà M, Escribano-Subías P, Martin C, Barberà JA, Cortina-Romero JM. Pulmonary thromboendarterectomy in chronic thromboembolic pulmonary hypertension: the Spanish experience. Ann Cardiothorac Surg 2022;11(2):151-160. doi: 10.21037/acs-2021-pte-18 thromboembolic pulmonary hypertension. Eur Respir Rev 2015;24:263-71.

24. Corsico AG, D'Armini AM, Cerveri I, et al. Long-term outcome after pulmonary endarterectomy. Am J Respir Crit Care Med 2008;178:419-24. 